\title{
Distributed Resource Allocation Based on Queue Balancing in Multi-hop Cognitive Radio Networks
}

\author{
Wei Wang ${ }^{\dagger}$, Kang G. Shin ${ }^{\ddagger}$ and Wenbo Wang ${ }^{\dagger}$ \\ ${ }^{\dagger}$ Wireless Signal Processing and Network Lab (WSPN) \\ Key Lab of Universal Wireless Communications, Ministry of Education \\ Beijing University of Posts and Telecommunications (BUPT), Beijing 100876, P.R. China \\ ${ }^{\ddagger}$ Real-Time Computing Laboratory (RTCL) \\ Department of Electrical Engineering and Computer Science \\ University of Michigan, Ann Arbor, MI 48109-2121, U.S.A. \\ Email: wwsunny@126.com,kgshin@eecs.umich.edu,wbwang@bupt.edu.cn
}

\begin{abstract}
Cognitive radio allows unlicensed users to access the licensed spectrum opportunistically to enhance the spectrum utilization efficiency. In this paper, the problem of allocating resources (channels and transmission power) in multi-hop cognitive radio networks (CRNs) is modeled as a multi-commodity flow problem with the dynamic link capacity resulting from dynamic resource allocation, which is in sharp contrast with existing flow control approaches that assume fixed link capacity. Based on queue-balancing network flow control that is suitable for handling dynamically changing spectrum availability in CRNs, we propose a distributed scheme (installed and operational in each node) for optimal resource allocation without exchanging spectrum dynamics information between nodes. Considering the power masks, each node makes resource-allocation decisions based on current or past information from neighboring nodes to satisfy the throughput requirement of each flow. Parameters of these proposed schemes are configured to maintain the network stability. The performance of the proposed scheme for asynchronous and synchronous scenarios is analyzed comparatively. We considered both cases of sufficient and insufficient network capacity.
\end{abstract}

\section{INTRODUCTION}

Cognitive radio (CR) [1]-[4] is an emerging technology for future wireless communication and networking. CR makes it possible for unlicensed/cognitive users to opportunistically utilize the licensed spectrum when it is not occupied by licensed/primary users. It can overcome the drawback of the current static spectrum allocation policy and improve the spectrum utilization.

For multi-hop wireless networks, cross-layer resource allocation [5][6] is a challenging problem; especially, distributed resource allocation is a very hard problem. Joint channel allocation, power control, route selection and congestion control, which affect one another, make the problem even more difficult. In this paper, flow control [7] (instead of routing) is adopted for transmission of data from a source to the corresponding destination.

For CR networks, each node has the power mask on every channel to protect primary users, and the spectrum status of channels in a licensed spectrum may change because of the primary users' activities, which is known as spectrum dynamics. These characteristics of CR networks introduce new challenges for resource allocation. There have been a number of publications on spectrum allocation [8][9], power control [10][11], and routing [12][13] for CR networks. However, most of them focus on one of the various aspects of resources allocation, and almost all of existing research on distributed resource allocation requires spread the spectrum dynamics information to the nodes all over the CR network.

In this paper, we propose a distributed resource allocation scheme that meets end-to-end (E2E) throughput demands for multiple sessions in multi-hop CR networks. The proposed scheme considers the power masks for each channel, and can adjust itself adaptively to accommodate the spectrum dynamics according to the spectrum status and the current link transmission requirement. The proposed distributed scheme is also suitable for asynchronous scenarios, in which all the nodes do not have to execute the scheme at the same time. The main contributions of this paper are summarized as follows: it

- Extends the queue-balancing flow control from wired networks with fixed link capacity to wireless networks in which link capacities are dictated by dynamic resource allocation. Especially, for CR networks this scheme is suitable for handling spectrum dynamics in a distributed manner.

- Proposes a node-level resource-allocation scheme deployed in each node based only on local information available to the node. The data rate, power level and channel allocation are determined by the current queue size and adjusted to accommodate the status of channels and the throughput requirement on each link.

- Investigate the network-level performance. The resultant parameter configurations guarantee the network stability if the network has large enough capacity to satisfy the throughput requirements of all sessions. The performance of the proposed scheme in asynchronous scenarios is analyzed and compared with that when all the nodes can execute the algorithm at the same time (i.e., synchronously). The case of insufficient network capacity is also considered.

The rest of this paper is organized as follows. Section II 
describes the system model. Section III applies the queuebalancing flow control to CR networks. Section IV analyzes the distributed resource allocation problem by joint rate control, power and channel allocation. Section V investigates the network performance of the proposed scheme with appropriate parameters. The practical issues are discussed further in Section VI. The related work and the conclusions are presented in Section VII and Section VIII, respectively.

\section{System Model}

The multi-hop CR network under consideration is assumed to consist of a set of nodes $\mathscr{V}$ and a set of links $\mathscr{L}$. Let $T(l)$ and $R(l)$ denote the transmitter and receiver of link $l$, respectively.

Each node is equipped with two radio interfaces, one for transmitting data and the other for receiving data. OFDM is assumed to have been deployed in the network, so that multiple channels can be used in each interface. In order to reduce the complexity of resource allocation, several subcarriers are combined to be a "channel." The authors of [5] provided a method for estimating the minimum number of channels required for wireless networks. Due to the different properties of channels in CR networks, the spectrum can be divided into more channels than that in [5] for flexibility. We will not focus on how to divide the channels, but assume that a set $\mathscr{K}$ of available channels are given. ${ }^{1}$

Note that the resource allocation and flow control in this paper are only for data transmission, not for control information. We assume that there are some other dedicated channels deployed for control information.

\section{A. Interference-aware Transmission Model}

Because of equipment's limited capability, each node has a power constraint $\overline{P_{i}}$, so the total power on all the channels should not exceed $\overline{P_{i}}$.

$$
\sum_{T(l)=i} \sum_{k \in \mathscr{K}} \omega_{l k} P_{l k}<\overline{P_{i}}
$$

where $P_{l k}$ is the transmit power at link $l$ on channel $k, \omega_{l k}$ is a binary indicator for channel allocation. $\omega_{l k}=1(0)$ means that channel $k$ is (not) allocated to link $l$.

Let $\mathscr{I}(l)$ be the set of links that interfere with link $l$. To avoid the interference between links, $\forall j \in \mathscr{I}(l), \omega_{l k}+\omega_{j k} \leq$ 1 . The set of interfering links can be constructed by either the protocol model based on distance, or the signal to interference and noise ratio (SINR) threshold model based on the required SINR [14].

Based on the Shannon capacity formula, the capacity of link $l$ can be written as

$$
C_{l}=\sum_{k \in \mathscr{K}} W_{k} \log \left(1+\frac{\omega_{l k} P_{l k} G_{l}}{I_{R(l) k}}\right)
$$

where $W_{k}$ is the bandwidth of channel $k, G_{l}$ is the path gain for link $l, I_{R(l) k}$ includes the thermal noise and the interference from the primary users and other systems.

\footnotetext{
${ }^{1}$ The subcarriers within a channel are considered homogeneous.
}

To protect the communication of the primary nodes, the transmit power of cognitive radio nodes should be restricted.

$$
\sum_{T(l)=i} \omega_{l k} P_{l k}<Q_{i k}
$$

where $Q_{i k}$ is the maximum transmit power of node $i$ on channel $k$.

$$
Q_{i k}= \begin{cases}Q_{i k}^{H} & \text { if no primary user } \\ Q_{i k}^{L} & \text { if primary user }\end{cases}
$$

If there is no primary node nearby, the CR nodes can transmit with as much power as they can. $Q_{i k}^{H}$ is the maximum allowed power for node $i$ on channel $k$ because of the equipment's limited capability. If some primary users are discovered via spectrum sensing, the CR nodes should transmit with the power less than a certain threshold $Q_{i k}^{L}$ to avoid an unacceptable level of interference to the primary receivers. Most of previous work [8][12] considered the binary model of channel availability for cognitive radios, which is a special case of our model if $Q_{i k}^{L}$ is set to 0 .

Besides the power mask for protecting the primary users/nodes, another characteristic of CR networks is the spectrum dynamics. The activities of primary nodes affect the channel capacities greatly for CR nodes.

\section{B. Traffic Flows with Required Throughput}

We would like to allocate resources (channels and transmit power) to meet the required throughput for each session. There are a set of traffic sessions $\mathscr{F}$ in the network. Each session $f$ is defined by a source node $S(f)$, a destination node $D(f)$, and the E2E throughput demand $r_{f}$. The total throughput over link $l$ must satisfy

$$
\sum_{f \in \mathscr{F}} x_{l f} \leq C_{l}
$$

where $x_{l f}$ is the data rate of session $f$ on link $l$ and $C_{l}$ is as defined in Eq. (2).

¿From the network flow's perspective, the flow conservation constraints need to be satisfied:

$$
\begin{gathered}
\sum_{T(l)=S(f)} x_{l f}=r_{f} \\
\sum_{T(l)=D(f)} x_{l f}=0 \\
\sum_{T(l)=i} x_{l f}=\sum_{R(l)=i} x_{l f} \quad(i \neq S(f), D(f)) .
\end{gathered}
$$

Note, however, that our problem doesn't satisfy the flow conservation constraints in a strict sense. Each node has a buffer to store the data to be forwarded, so it doesn't have to satisfy the conservation constraints over a short time duration, but must satisfy them in a long-time average sense. 


\section{Queue-Balancing For Network Flow Control}

According to the problem formulation, the problem can be modeled as a multi-commodity flow problem. The multicommodity flow problem is commonly solved by pricedirective decomposition and the resource-directive decomposition [7]. These methods divide the multi-commodity flow problem into single-commodity flow problems and find the paths for each commodity. However, they require a centralized control. Because link capacities are not fixed in our problem setting, but determined by the resource allocation in wireless networks, the nodes should be synchronized to adjust the flow and link capacity. The authors of [15] and [16] proposed another method, called queue-balancing flow control, for the multi-commodity flow problem. It does not choose paths for each commodity, but pushes the data from sources to the corresponding destinations by using "queue potential." Fig. 1 shows a simple example of queue-balancing flow control.

In order to meet the throughput demands of every session/flow in the network, a dynamic (instead of static) queuebalancing algorithm is used for the multi-commodity flow problem. There is a queue for each session at both the transmitter and the receiver of each link, as shown in the right-below subfigure of Fig. 1. The traffic of the sessions enters the network from the sources and exits the network from the destinations. The objective of resource allocation is to maximize the total potential decrease for transmitting the data.

Because data queuing lets some data remain in the network, to meet the data-rate (throughput) requirement, data needs to be pumped into the network at a rate higher than the required rate. Therefore, the data enters the network at the rate $\{(1+$ $\left.\epsilon) r_{f}\right\}$.

The potential function of a queue which belongs to session $f$ of size $q$ is set to the same as that in [16].

$$
\phi_{f}(q)=e^{\alpha_{f} q} .
$$

If the queue of some source $S(f)$ is beyond $q_{f}^{\max }$, the overtop data is stored at another special overflow buffer. The potential function of an overflow queue of session $f$ of size $b_{f}$ is $b_{f}$. $\phi_{f}^{\prime}\left(q_{f}^{\max }\right)$.

This type of parameter configuration guarantees the stability of a distributed scheme as long as the network capacity is large enough to meet the throughput demands of all sessions, which will be detailed in Section V.

The queue-balancing flow control in this paper is similar to that in [16], but the latter cannot be applied to multi-hop CR networks directly. Some nontrivial changes are necessary for the following reasons. First, the link capacities change with dynamic resource allocations. Second, in multi-hop wireless networks, it is difficult to synchronously execute a distributed algorithm at different nodes. The parameters should be configured appropriately to guarantee the network stability while considering asynchronous scenarios. Third, additional coordination of nodes is needed because of the complex wireless environment, especially caused by primary users moving in and out of channels.

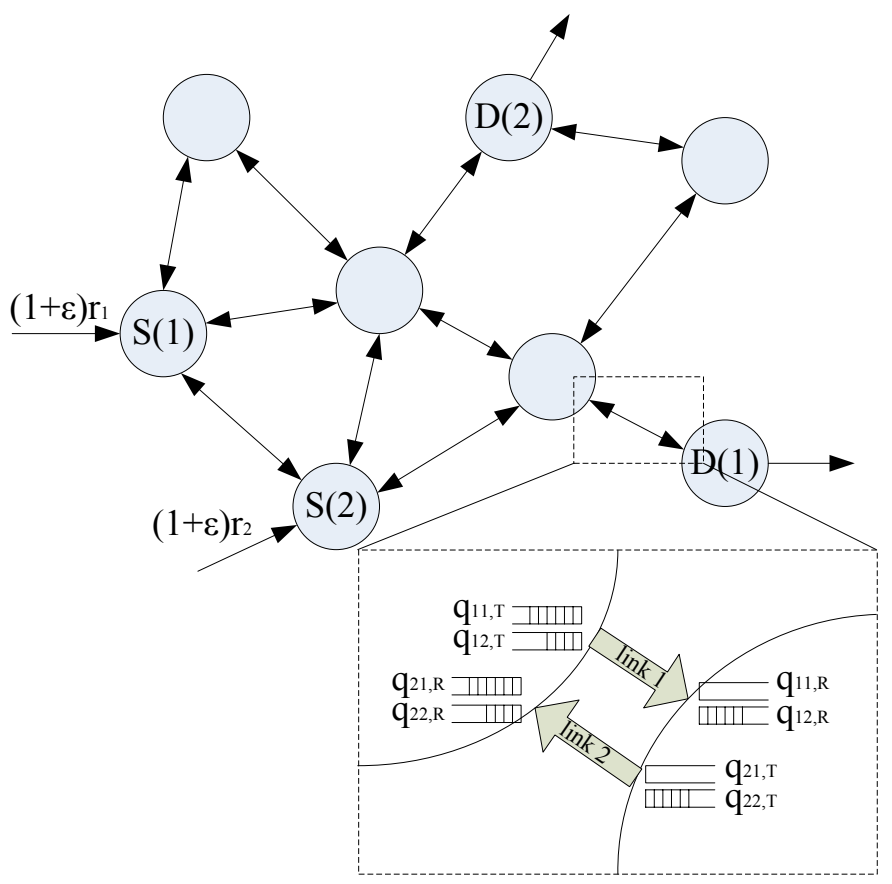

Fig. 1. An illustration for queue-balancing flow control

\section{Node-LeVel Resource Allocation}

By adopting the queue-balance flow control, the problem can be transformed to a resource-allocation problem for each link. Specifically, we need to allocate the channel and power resource for each link so as to maximize the decrease of potential functions in each time slot. ${ }^{2}$ The potential decrease is expressed as

$$
\begin{array}{r}
\delta_{l f}=\phi_{f}\left(q_{l f, T}\right)-\phi_{f}\left(q_{l f, T}-x_{l f}\right) \\
+\phi_{f}\left(q_{l f, R}\right)-\phi_{f}\left(q_{l f, R}+x_{l f}\right)
\end{array}
$$

where $q_{l f, T}$ and $q_{l f, R}$ are the queue size of link $l$ and session $f$ at the transmitter and the receiver, respectively. This objective function balances the size of queues. Because of the exponential potential function, the network provides higher priority to those sessions with large queue sizes and larger size differences between queues of the transmitter and the receiver of a link.

For allocating resources to maximize the potential decrease, we consider three types of resource allocation, ranging from small-scale to larger-scale adjustments.

- Adjust the data rate over a link for each session according to the queue sizes for each session at transmitters and receivers.

- Allocate the power of the links transmitting from the same nodes. The power adjustment changes the link capacities, but doesnot affect other links because of interference-free channel allocation.

\footnotetext{
${ }^{2}$ Consider the time interval of a time slot as a unit of time, so it is omitted in the rest of this paper for simplicity of expression.
} 
- Change the channel allocation to achieve better performance. To avoid the interference between links, nearby nodes need to be coordinated for channel allocation.

\section{A. Rate Control over a Wireless Link}

For a link with fixed capacity, the problem is how to allocate the total capacity to the sessions that go through this link, such that the total decrease of potential is maximized. The total potential decrease over link $l$ is

$$
\delta_{L_{l}}=\sum_{f \in \mathscr{F}(l)} \delta_{l f}
$$

where $\mathscr{F}(l)$ is the set of sessions that go through link $l$.

The problem is to maximize the total potential decrease $\delta_{L_{l}}$ subject to the link-capacity constraint Eq. (5). The linkcapacity constraint is a complicating bundle constraint for all the sessions over this link. We decompose this multiple session rate-control problem into multiple single-session rate-control problems by placing cost on the constraint with a Lagrangian multiplier.

$$
\Delta_{l}=\sum_{f \in \mathscr{F}(l)} \delta_{l f}-\lambda_{l}\left(\sum_{f \in \mathscr{F}(l)} x_{l f}-C_{l}\right)
$$

where $\lambda_{l}$ is the Lagrangian multiplier for link $l$.

By decomposition, for each session $f$, the objective of flow control is to maximize the decomposed Lagrangian function.

$$
\Delta_{l f}=\delta_{l f}-\lambda_{l} x_{l f}
$$

where the optimal value of $\lambda_{l}$ is the one that satisfies $\sum_{f \in \mathscr{F}(l)} x_{l f}=C_{l}$.

The maximum potential decrease is achieved when the firstorder partial derivative of $\Delta_{l f}$ with respect to $x_{l f}$ is set to 0 .

$\frac{\partial \Delta_{l f}}{\partial x_{l f}}=\alpha_{f} e^{\alpha_{f}\left(q_{l f, T}-x_{l f}\right)}-\alpha_{f} e^{\alpha_{f}\left(q_{l f, R}+x_{l f}\right)}-\lambda_{l}=0$.

The data rate of each session on link $l$ can be calculated as

$$
x_{l f}^{*}=\frac{1}{\alpha_{f}} \ln \frac{\sqrt{\left(\frac{\lambda_{l}}{\alpha_{f}}\right)^{2}+4 e^{\alpha_{f}\left(q_{l f, R}+q_{l f, T}\right)}}-\frac{\lambda_{l}}{\alpha_{f}}}{2 e^{\alpha_{f} q_{l f, R}}} .
$$

Especially, for the problem with large enough link capacity, the capacity constraint is not tight. For each session $f$, the optimal data rate is

$$
x_{l f}^{*}=\frac{q_{l f, T}-q_{l f, R}}{2}
$$

\section{B. Power Allocation to Nodes}

The total potential decrease of the links that are used to transmit data from node $i$ is

$$
\delta_{N_{i}}=\sum_{T(l)=i} \delta_{L_{l}}=\sum_{T(l)=i} \sum_{f \in \mathscr{F}(l)} \delta_{l f} .
$$

Considering the effect of power allocation for each link $l$ and each channel $k$ on the total potential decrease for node $i$, the partial derivative is

$$
\frac{\partial \delta_{N_{i}}}{\partial P_{l k}}=\frac{\partial \delta_{L_{l}}}{\partial C_{l}} \frac{\partial C_{l}}{\partial P_{l k}}
$$

Obviously, a larger potential decrease on channel $k$ over link $l$ can be achieved with a larger power $P_{l k}$. However, the transmit power is limited by the equipment's capability. How to allocate the limited power $P_{i}$ of node $i$ to every channel of every link transporting data from this node is the problem we want to solve.

The power allocation can be divided into two levels. First, we consider the power allocation to the channels $\mathscr{K}(l)$ with a given link power $P_{l}$. Then, based on the analysis considering a given link power, we allocate the node power to the links to maximize the total potential decrease $\delta_{N_{i}}$.

The water-filling allocation between the channels within a link can achieve the optimal capacity, even if there are power masks for the channels in cognitive radio networks.

Theorem 1: For a given set $\mathscr{K}(l)$ of channels and allocated power $P_{l}$ on link $l$, the water-filling power allocation can achieve the optimal performance. Let $\Psi_{l}$ be the water-filling level of link $l$, then the power should be allocated as

$$
P_{l k}=\min \left\{\left[W_{k} \Psi_{l}-I_{R(l) k} / G_{l}\right]^{+}, Q_{T(l) k}\right\}
$$

where $[a]^{+}=\max \{0, a\}$, and the water-filling level $\Psi_{l}$ is set to satisfy

$$
\sum_{k \in \mathscr{K}(l)} P_{l k}=P_{l}
$$

Proof: Considering only the total power constraint for this link $\sum_{k \in \mathscr{K}(l)} P_{l k} \leq P_{l}$, the optimal solution can be obtained by the Lagrange multiplier method, which is similar to the proof in [18].

$$
P_{l k}=\left[W_{k} \Psi_{l}-I_{R(l) k} / G_{l}\right]^{+} .
$$

The partial derivative of $C_{l}$ with respect to $P_{l k}$ can be calculated from Eq. (2).

$$
\frac{\partial C_{l}}{\partial P_{l k}}=\frac{W_{k} G_{l}}{P_{l k} G_{l}+I_{R(l) k}} .
$$

It is obvious from Eq. (22) that the derivative is non-negative, meaning that $C_{l}$ will not decrease with the increase of $P_{l k}$. For the total link-power constraint and the power mask constraints for each channel, it is necessary for the optimal solution that at least one of the constraints is tight for each channel. Therefore, the optimal solution is to allocate the maximum power subject to two constraints, and Eq. (19) follows.

Based on the water-filling power allocation between the channels within a link, each channel belongs to one of three types of states, as shown in Fig. 2.

- State 1: No power is allocated because of large $I_{R(l) k} / G_{l}$,

$$
W_{k} \Psi_{l}-I_{R(l) k} / G_{l} \leq 0
$$

- State 2: Power is allocated according to the water-filling level but not the power mask,

$$
0<W_{k} \Psi_{l}-I_{R(l) k} / G_{l}<Q_{T(l) k}
$$

- State 3: Power is allocated according to the power mask.

$$
W_{k} \Psi_{l}-I_{R(l) k} / G_{l} \geq Q_{T(l) k}
$$




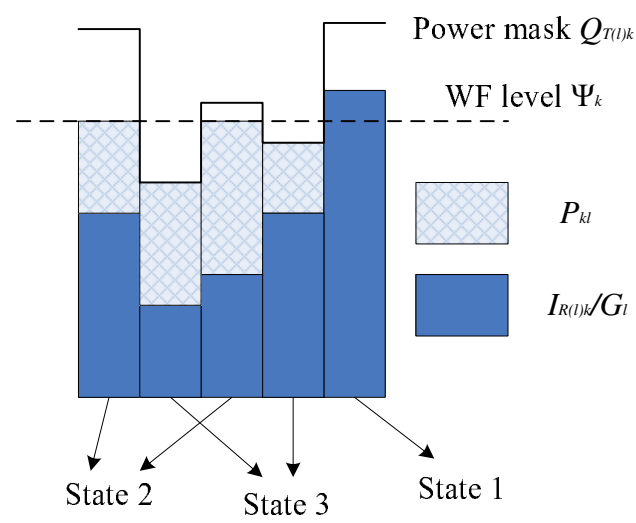

Fig. 2. Three states for power allocation between the channels within a link

Let $\mathscr{K}_{l}^{\text {act }}$ be the set of channels in State 2 whose power is determined by the water-filling level for link $l$. The capacities of the channels in $\mathscr{K}_{l}^{a c t}$ change when the link power $P_{l}$ changes. According to Theorem 1, the partial derivative of $C_{l}$ with respect to the allocated power $P_{l}$ for link $l$ is

$$
\frac{\partial C_{l}}{\partial P_{l}}=\frac{1}{\Psi_{l} \sum_{k \in \mathscr{K}_{l}^{a c t}} W_{k}} .
$$

For the link power allocation, Eq. (18) can be transformed as

$$
\frac{\partial \delta_{N_{i}}}{\partial P_{l}}=\frac{\partial \delta_{L_{l}}}{\partial P_{l}}=\frac{\partial \delta_{L_{l}}}{\partial C_{l}} \frac{\partial C_{l}}{\partial P_{l}}
$$

$\partial \delta_{L_{l}} / \partial C_{l}$ is just the Lagrangian multiplier $\lambda_{l}$ in the last subsection, and $\partial C_{l} / \partial P_{l}$ is given by Eq. (23). $\partial \delta_{L_{l}} / \partial P_{l}$ is non-negative and decreases with the increase of $P_{l}$. To achieve the maximum potential decrease $\delta_{N_{i}}$, the optimal method for power allocation between links is to allocate the node power to the links such that the derivative $\partial \delta_{L_{l}} / \partial P_{l}$ is equal for all the links transmitting data from the same node.

\section{Interference-free Channel Allocation}

1) Effect of Changing Channel Occupation: In order to allocate channels efficiently, we first estimate the potential change as a result of adding or subtracting a channel for each link. Let $C_{l, k}$ be the capacity of link $l$ when the set of channels on link $l$ is $\mathscr{K}(l) \backslash k$ if $k \in \mathscr{K}(l)$, or $\mathscr{K}(l) \bigcup k$ if $k \notin \mathscr{K}(l)$. Using the water-filling power allocation, we can obtain $C_{l, k}$. Let $\delta_{L_{l}}^{*}(C)$ denote the optimal potential decrease over link $l$ achieved by calculating $x_{l f}$ as Eq. (15) when the link capacity is $C$. Finding an optimal $\lambda_{l, k}$, which is the Lagrangian multiplier for the case when $\omega_{l k}$ changes, we can calculate $\delta_{L_{l}}^{*}\left(C_{l, k}\right)$. This way, the change of the potential decrease is $\left|\delta_{L_{l}}^{*}\left(C_{l, k}\right)-\delta_{L_{l}}^{*}\left(C_{l}\right)\right|$.

However, $\delta_{l}^{*}\left(C_{l, k}\right)$ indicates the potential change, only considering the allocated power for link $l$ fixed at $P_{l}$. By having the transmitter nodes adjust the power allocation adaptively, the potential decrease can be improved. It is difficult to calculate the exact improvement of the potential decrease, because the channel allocation on other links of this node may also change. Let $N_{i}^{i n}$ be the number of incoming links and
$N_{i}^{\text {out }}$ the number of outgoing links of node $i$. We can then estimate the change of the potential decrease as

$$
\left|\frac{\partial \delta_{L_{l}}^{*}\left(C_{l}\right)}{\partial p_{l}}-\frac{\partial \delta_{L_{l}}^{*}\left(C_{l, k}\right)}{\partial p_{l}}\right| \frac{N_{T(l)}^{\text {out }}}{N_{T(l)}^{\text {out }}+1} .
$$

The above expression represents the effect of adaptive power allocation between the links that have the same transmitter node as link $l$. Based on the analysis in the last subsection, $\partial \delta_{L_{l}}^{*}\left(C_{l}\right) / \partial p_{l}$ is the same for all the links of a node. If the channel allocation causes a larger difference between $\partial \delta_{L_{l}}^{*}\left(C_{l, k}\right) / \partial p_{l}$ and $\partial \delta_{L_{l}}^{*}\left(C_{l}\right) / \partial p_{l}$, the adaptive link power allocation can make a larger improvement. On the other hand, the improvement is larger if the node has more outgoing links, because the power allocation between links has more degrees of freedom.

It is defined in the system model that a set of links $\mathscr{I}(l)$ have conflict with link $l$. We also define $\mathscr{N}\left(L_{l}\right)$ as the nodes that are the transmitters of the links in $\mathscr{I}(l)$, and $\mathscr{N}\left(N_{i}\right)$ as the set of nodes within the interfering range of node $i$. The number of the interfering links $\mathscr{I}(l)$ of link $l$ is

$$
N_{l}^{I}=N_{T(l)}^{i n}+N_{R(l)}^{\text {out }}+\sum_{i \in \mathscr{N}(T(l))} N_{i}^{\text {in }}+\sum_{i \in \mathscr{N}(R(l))} N_{i}^{\text {out }}-1 .
$$

The first two terms are to guarantee that the channel is used for at most one of the node's links. For this purpose, besides the two terms, there are also the links transmitting from $T(l)$ and the links receiving at $R(l)$, which are included in the third and fourth terms.

Because the allocation of a channel for link $l$ means that other $N_{l}^{I}-1$ links can't use this channel. Although this channel may achieve different performance on all the interfering links because of different power masks, the utility divided by $N_{l}^{I}$ can give an estimation of potential change in terms of the average of all the interfering links.

Let $U_{l k}$ denote the achieved utility on the potential if link $l$ is assigned channel $k$. Considering both the change of the potential decrease with fixed link power and the effect of adaptive link power allocation, if $k \notin \mathscr{K}(l)$,

$$
U_{l k}=\frac{N_{T(l)}^{\text {out }}\left(\delta_{L_{l}}^{*}\left(C_{l, k}\right)-\delta_{L_{l}}^{*}\left(C_{l}\right)\right)\left|\frac{\partial \delta_{L_{l}}^{*}\left(C_{l}\right)}{\partial p_{l}}-\frac{\partial \delta_{L_{l}}^{*}\left(C_{l, k}\right)}{\partial p_{l}}\right|}{N_{l}^{I}\left(N_{T(l)}^{\text {out }}+1\right)} .
$$

If $k \in \mathscr{K}(l)$,

$$
U_{l k}=\frac{\left(N_{T(l)}^{\text {out }}+1\right)\left(\delta_{l}^{*}\left(C_{l}\right)-\delta_{l}^{*}\left(C_{l, k}\right)\right)}{N_{T(l)}^{\text {out }} N_{l}^{I}\left|\frac{\partial \delta_{L_{l}}^{*}\left(C_{l}\right)}{\partial p_{l}}-\frac{\partial \delta_{L_{l}}^{*}\left(C_{l, k}\right)}{\partial p_{l}}\right| .}
$$

2) Coordination Between Links: When channel allocation to links is optimal for each channel, so is the channel allocation in the network. We can thus consider channels individually by restricting at most one channel change on a link at a time.

Allocation of a single channel can be modeled as a weighted independent set problem, which is NP Complete [19]. So, we propose a greedy channel-allocation protocol, achieving suboptimality. 
We define three types of messages, INFO, REQ, OCCUPY, which contain the information of channel index, link index, and the corresponding channel-allocation utility. Note that for a given link $l$, messages are sent to the transmitters $\mathscr{N}\left(L_{l}\right)$ of all the links that interfere with link $l$. For simplicity, the messages can be sent to the nodes within two times of the interfering range from node $T(l)$, such that all the nodes in $\mathscr{N}\left(L_{l}\right)$ can receive the messages. The following part describes the information exchange process at link $l_{0}$ for allocating channel $k_{0}$.

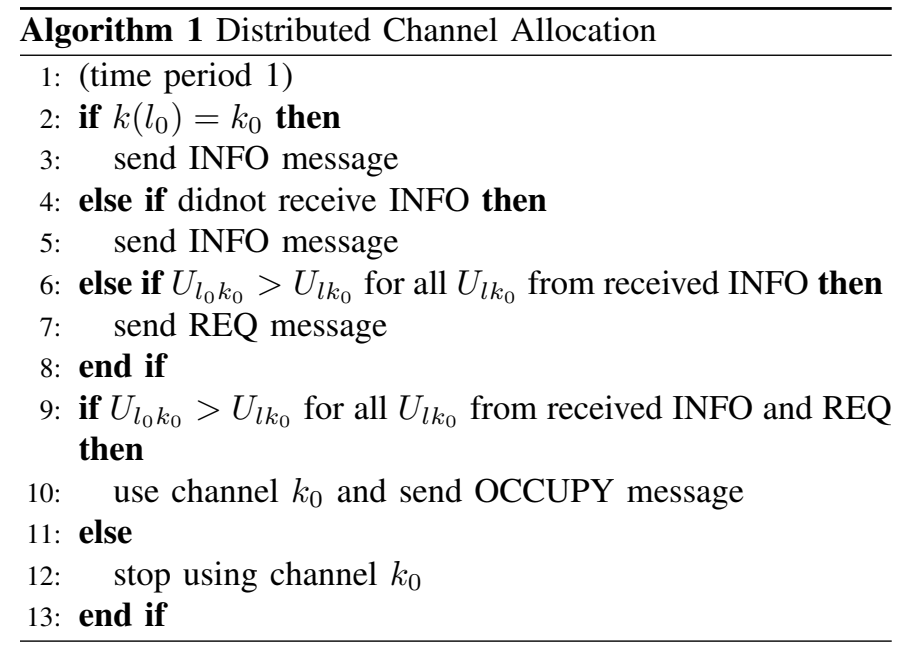

Theorem 2: The proposed distributed coordination of channel allocation yields interference-free channel allocations.

Proof: Consider only one channel $k$ in this proof. Suppose there exist two links $l$ and $m$ which interfere with each other. Without loss of generality, $U_{l k}>U_{m k}$. If link $l$ sends INFO or REQ messages, then the channel will not be allocated to link $m$. If channel $k$ is allocated to link $m$, link $l$ should send neither INFO nor REQ messages. As the proposed coordination scheme, link $l$ does not transmit INFO messages only if link $l$ interferes with another link $j$, and link $l$ does not transmit REQ messages only if $U_{l k}<U_{j k}$ or link $l$ has requested the allocation of another channel. In such cases, the channel will not be allocated to link $l$.

Therefore, at most one of the interfering links can house the channel, so the channel allocation is interference-free.

By repeating the proposed distributed coordination of channel allocation until all the nodes either transmit or receive INFO messages during the first step, the resultant channel allocation is not optimal, but is a maximum set in the sense that the transmitting link set is not contained by any other transmitting set for each channel.

\section{Network-Level Resource Allocation}

\section{A. Node-based Distributed Algorithm}

Based on the above analysis, we propose a node-based distributed algorithm for joint flow control, channel allocation and power control.

Step 1:Adjust the queue sizes for each node.
Step 2:Broadcast the control information needed for resource allocation.

Step 3:Pre-determine the resource allocation and broadcast the INFO and REQ messages, if necessary.

Step 4:Determine the channel allocation strategy and the corresponding power and data rate for each session. Exchange OCCUPY messages.

In Step 1, add $(1+\epsilon) r_{f}$ to the queue size at the source nodes. At the destinations, decrease the size of the queues of the corresponding sessions to 0. Balance the queue sizes within each node for all of its sessions.

In Step 2, nodes transmit the control information to the nodes which transmit data to it. The information includes the noise and the inference from other systems $I_{i k}$ for each channel, the queue size for each session $q_{l f, R}$ for receiver links and the number of the transmitting links and the channelquality feedback $G_{l}$. In addition, the nodes need to transmit the link number $N_{i}^{i n}$ and $N_{i}^{\text {out }}$ to the nodes $\mathscr{N}(i)$.

In Step 3, we restrict at most one REQ message transmitted for each link, because the channel occupation utilities are calculated by considering only one channel allocation change based on the current status, although channels are allocated individually as described in the last section. Channel $k$ is chosen if the difference between $U_{l k}$ and the maximum utility of the received messages is the largest for all channels.

In Step 4, the channel allocation is determined according to the INFO and REQ messages. Based on the allocated channels for each link, the optimal power allocation for the links and the rate control scheme within each link are deployed as described in Section IV.A and IV.B.

\section{B. Performance}

In order to evaluate the performance of the proposed algorithms, an ad-hoc secondary network is employed for dynamic simulation in multi-hop CR networks. 20 secondary users are distributed randomly in a $30 \mathrm{~km} \times 30 \mathrm{~km}$ area, and 4 sessions in this network have the data rate $2 \mathrm{Mbps}$, $5 \mathrm{Mbps}$, $8 \mathrm{Mbps}$ and $10 \mathrm{Mbps}$, respectively. The parameter $\epsilon$ in queue balancing scheme is set to 0.2. 10 channels are considered, each of which has a bandwidth of $5 \mathrm{MHz}$.

The path loss is calculated based on the distance using a free space propagation model as $P L=P L_{0} \cdot d / d_{0}$, where $P L_{0}=70 \mathrm{~dB}$ and $d_{0}=1 \mathrm{~km}$. The maximum transmit power is $10 \mathrm{dBm}$ and the thermal noise power is $-100 \mathrm{dBm}$. Each primary user occupies a channel randomly. To protect the primary users, the power masks are set to guarantee the interference at the primary users to be less than $-100 \mathrm{dBm}$.

Figs. 3 and 4 show the stability of queue size and E2E throughput, respectively, when there is sufficient network capacity. When a new session begins, a period of time is needed to spread packets to the nodes all over the network to build the queue system. After this period, the size of queues would not always increase and the required throughput can be achieved if the network capacity is large enough. The condition for network stability is provided in [16]. It can also be observed that the curves converge after 1500 time slots 


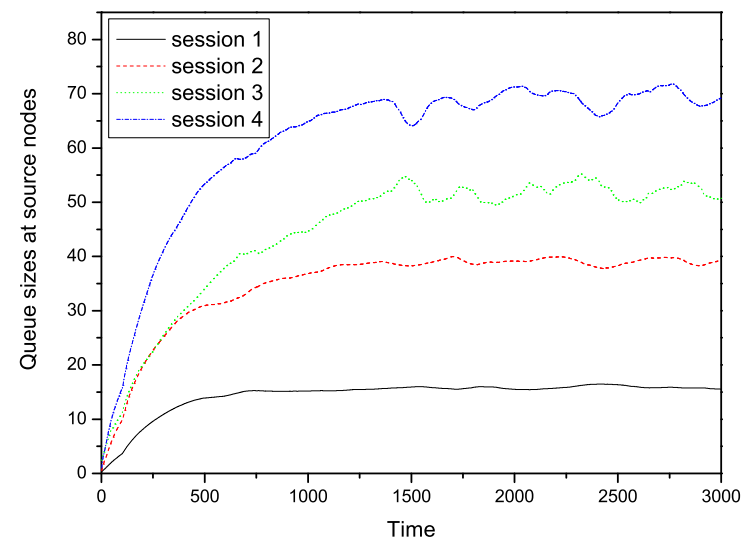

Fig. 3. Stability of the queue sizes of source nodes

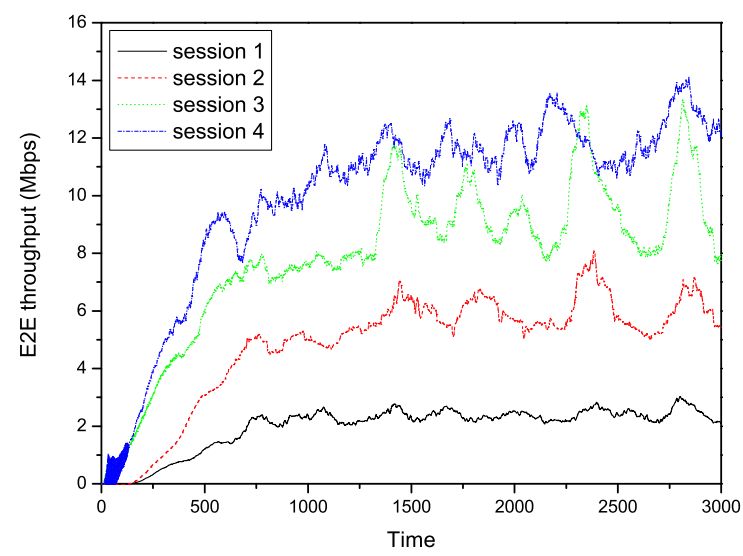

Fig. 4. Stability of E2E throughput

in this configuration. Therefore, we use the performance after 1500 time slots as steady-state performance. Note that we use the average value within a 100 time slot window rather than the value just at this time slot.

Fig. 5 presents the total E2E throughput performance of the proposed scheme. The random spectrum allocation and average power \& rate allocation are considered as the baselines for the comparison. The result shows the improvement of the proposed scheme.

Fig. 6 shows the E2E throughput with a different number of primary users. With more primary users, the transmit powers of secondary users are limited more strictly, and less network capacity is achieved. When there are 10 channels, the proposed scheme can guarantee the required throughput of all sessions even if there are 10 primary users. With larger number of primary users, the proposed scheme can achieve more improvement, because it can allocate the channels to the secondary links which is far away from the primary users occupying them.

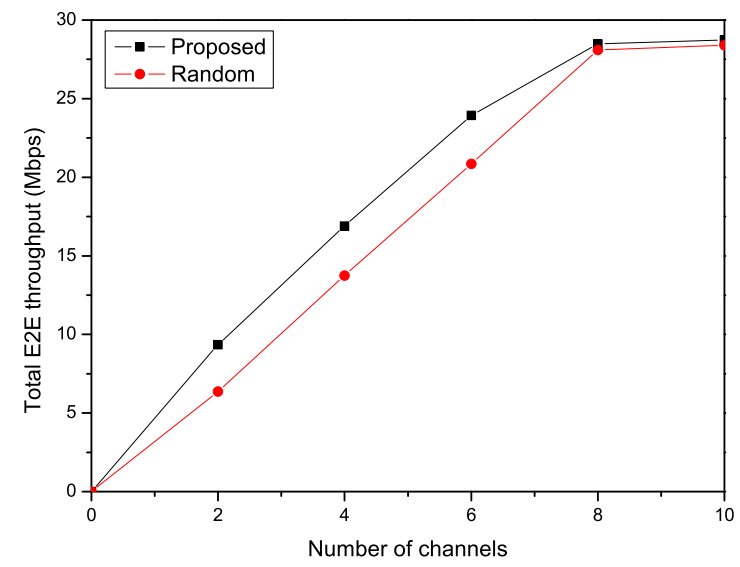

Fig. 5. Total E2E throughput v.s. number of channels

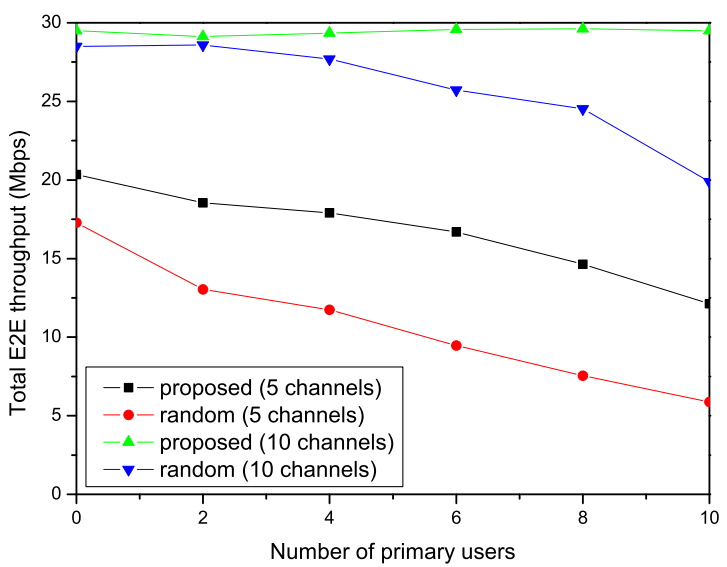

Fig. 6. Total E2E throughput v.s. number of primary users

\section{Asynchronous Scenarios}

So far, the proposed distributed scheme has been based on an assumption that all the nodes execute this procedure at the same time. We need to consider the case when execution of the resource allocation procedure is not synchronized (i.e., asynchronous scenarios). In such a case, our algorithm in each node is similar to that in the synchronous case, but uses the information about other nodes last time when they made resource-allocation decisions. This use of "old" information has the following effects.

- The queue size information is not accurate, which may cause some error in allocating resources to maximize the potential decrease.

- Nodes have to wait for one period when they want to use a new channel such that all the other interfering nodes stop using this channel.

1) Inaccurate Queue Size Information: If we use the queuesize information received during the last period, the actual potential decrease may not be the same as the estimated value. For the obsolete queue-size information of receivers, if the queue size received last time is larger than the current actual 
queue size, the estimated potential decrease is larger than that of using current information. In the worst case, no data is transmitted out of $R(l)$ and the queues at the transmitters of all the links whose receiver is $R(l)$ have the largest queue size $q_{f}^{\max }$. The maximum possible queue size of receiving links at $R(l)$ is $\frac{q_{f}^{\max }+q_{l f, R}}{2}$ because of Eq. (16). The numbers of the queues for transmission and reception links in a node are the same. So, the actual queue size is at most

$$
\frac{\frac{q_{f}^{\max }+q_{l f, R}}{2}+q_{l f, R}}{2} .
$$

The upper-bound error of the receiver's queue size is $\frac{q_{f}^{\max }-q_{l f, R}}{4}$.

If the queues in this network can grow unboundedly, the network becomes unstable. Let $L$ be the length of the longest flow path in the network and $|\mathscr{F}|$ be the number of sessions. Considering the error in queue-size information, an appropriate parameter configuration is needed to guarantee the network stability if the network transmission capacity is large enough.

Theorem 3: The network is stable using the proposed asynchronous scheme with

$$
\begin{gathered}
\alpha_{f}=\frac{\epsilon}{16 L r_{f} \ln \frac{4 L}{3 \epsilon}} \\
q_{f}^{\max }=\frac{1}{\alpha_{f}} \ln \left(\frac{2|\mathscr{F}|(1+2 \epsilon)}{\epsilon(1-2 \epsilon)}\right)
\end{gathered}
$$

if the network has the capacity to transmit $(1+2 \epsilon) r_{f}$ for each flow $f$.

\section{Proof: See Appendix A.}

¿From Theorem 3, we can see that $\alpha_{f}$ is smaller and $q_{f}^{\max }$ is larger than those in the synchronous case in [16]. Without synchronization, larger buffers are needed in network nodes, and more data may be queued up in the network.

2) Overhead of Channel Re-allocation: The other effect of asynchrony is the waiting for a channel re-allocation. If a link wants a new channel to be allocated, it has to wait for one time slot and then "grab" the channel in order to guarantee that all the interfering links have stopped using the channel, as shown in Fig. 7. Let $t_{l_{1} l_{2}}^{w}$ be the waiting time when link $l_{2}$ grabs the channel on link $l_{1}$. Considering the channel-switch overhead, if link $l_{2}$ wants to grab a new channel away from link $l_{1}$, the link $l_{2}$ 's channel accommodation utility $U_{l_{2} k}$ should be divided by $\left(1+t_{l_{1} l_{2}}^{w}\right)$ for normalization, and then compares it with $U_{l_{1} k}$.

During the waiting time, the channel switch will succeed if it gains a larger normalized utility from the messages received from others. However, it is possible that some larger accommodation utilities may appear during the waiting, such that the channel can't be used for a long time. To avoid this situation, if any of the links which are holding the channel stops using the channel, meaning that the channel is in the switching mode, the link stops transmitting REQ messages for this channel in one time slot even if it has a larger normalized channel holding utility. If an OCCUPY message from link $l$ was received in the last time slot but is not received in the
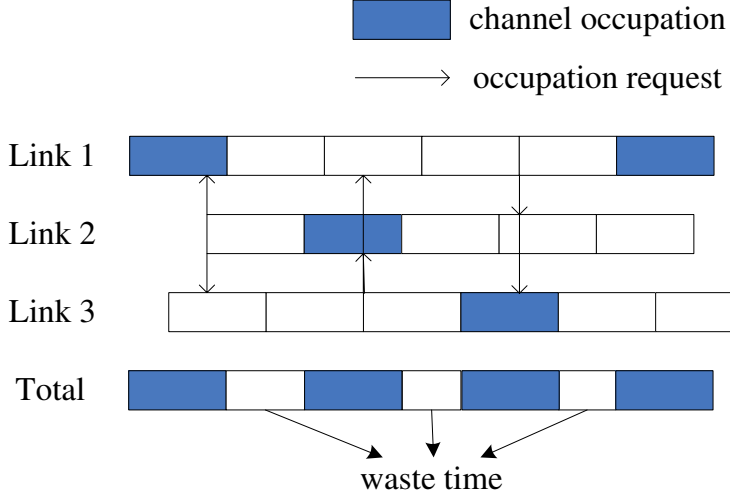

Fig. 7. An example asynchronous channel switch

current time slot, the node can know that link $l$ stopped using the channel.

Consider a link conflict graph, in which links are the vertexes and there are edges between the interfering links. The following theorem gives an upper bound of resource waste because of waiting for a channel switch.

Theorem 4: Let $N$ be the vertex number of the maximum clique in the link conflict graph, the portion of time wasted by the proposed scheme for the asynchronous case is at most $\frac{N-1}{2 N-1}$.

Proof: Any two vertices in a clique have an edge to connect with each other. The links in a clique interfere with each other and only one of them can transmit at a time.

Consider the smallest period from the time the channel is held by a link to the time that the channel returns to this link. No link uses the channel more than once, so this period is the smallest. The total waiting time in this period is

$$
t^{w}=t_{l_{1} l_{2}}^{w}+t_{l_{2} l_{3}}^{w}+t_{l_{3} l_{4}}^{w}+\ldots+t_{l_{(n-1)} l_{n}}^{w} .
$$

Because $t_{l_{i} l_{j}}^{w}+t_{l_{j} l_{i}}^{w}=T$,

$$
t^{w}=n T-\left(t_{l_{2} l_{1}}^{w}+t_{l_{3} l_{2}}^{w}+t_{l_{4} l_{3}}^{w}+\ldots+t_{l_{n} l_{(n-1)}}^{w}\right) .
$$

The second term of the right side of the above equation is just the waiting time the links hold the channel as the opposite sequence from $l_{n}$ to $l_{1}$, which is at least $T$ seconds, so $t^{w} \leq$ $(n-1) T$ and the portion of waiting time is at most $\frac{n-1}{n+(n-1)}$. For the clique with $N$ vertices, the waiting time portion is at most $\frac{N-1}{N+(N-1)}$.

In a graph containing a maximum clique of $N$ vertices, for the same period as the above analysis, the wasted time is also $(n-1) T$, but it is possible that more than one link hold the channel at the same time. Therefore, the waiting time is less than $\frac{N-1}{2 N-1}$.

Considering the link capacity at each time slot, the capacity loss during the waiting is less than that at the next time slot which has the largest channel holding utility in that slot.

Corollary 1: The average capacity in the asynchronous case is more than $1 / 2$ of that in the synchronous case. 


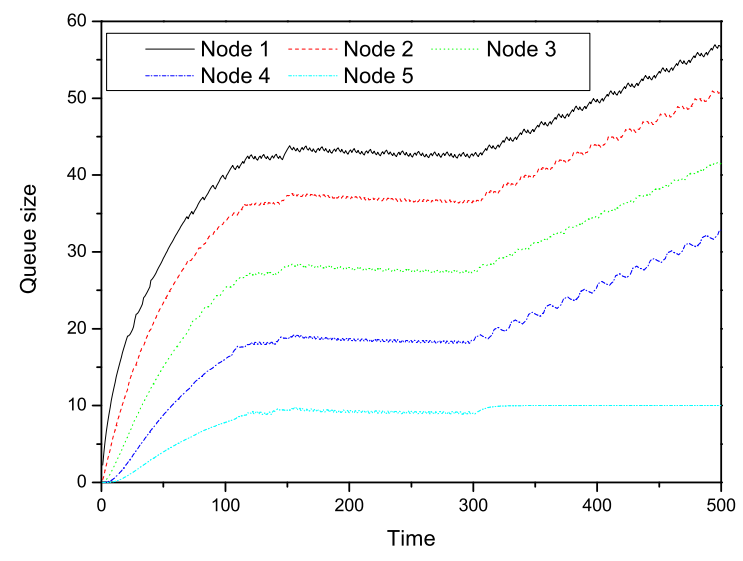

Fig. 8. Change of queue sizes for spectrum dynamics

\section{Spectrum Dynamics}

One of the advantages of the proposed scheme is the adaptation to spectrum dynamics. In multi-hop CR networks, because of the presence of primary users, the channel condition varies within the licensed spectrum. On the channels the primary users occupy, the nearby CR users cannot use the power beyond $Q_{i k}^{L}$ to protect the primary users' communication. Using the queue-balancing flow control, the algorithm need not control the data flow to the links with better channel situation when making a resource allocation, but only consider the queue sizes that are affected by the spectrum dynamics and represent the channel situation. The proposed scheme is more adaptive to spectrum dynamics and requires only local information, which is essential for distributed control.

The following theorem gives the requirement of the transmission capability of the network. In spite of the varying channel situation, as long as the average E2E throughput can meet the requirement, the network stability can be guaranteed.

Theorem 5: If the network can transmit more than $\frac{2+2 \epsilon}{2-\epsilon} r_{f}$ for each session $f$, then the network stability can be guaranteed by adjusting the configurable maximum queue size.

Proof: See Appendix B.

Fig. 8 gives an example for the change of queue size if the capacity of one of the links changes. Our simulation scenario is a simple 5-link chain, and the session flow goes from node 1 to node 6 . Each link has $10 \mathrm{Mbps}$ capacity, and the capacity of link 4 changes from $10 \mathrm{Mbps}$ to $5 \mathrm{Mbps}$ at time slot 300 . If the capacity of a link decreases, the queue sizes of the nearby nodes increase. This way, the resource allocation schemes at the nodes which are far away from the changed link can be adapted using the spectrum dynamics information without exchanging messages.

\section{E. Insufficient Network Capacity}

The analysis in this paper is based on the assumption that the network capacity is large enough for handling sessions'requirements. In case the throughput requirements of sessions are higher than the network capacity, admission

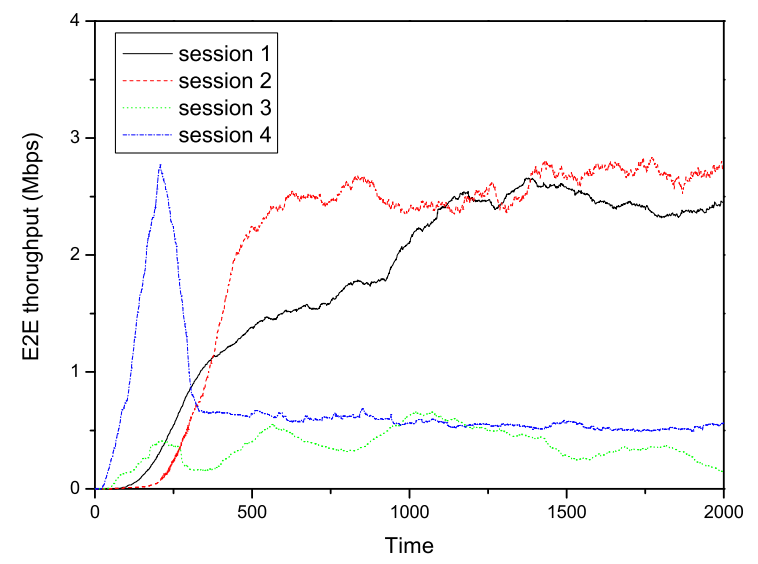

Fig. 9. E2E throughput in case of insufficient network capacity without any drop mechanism

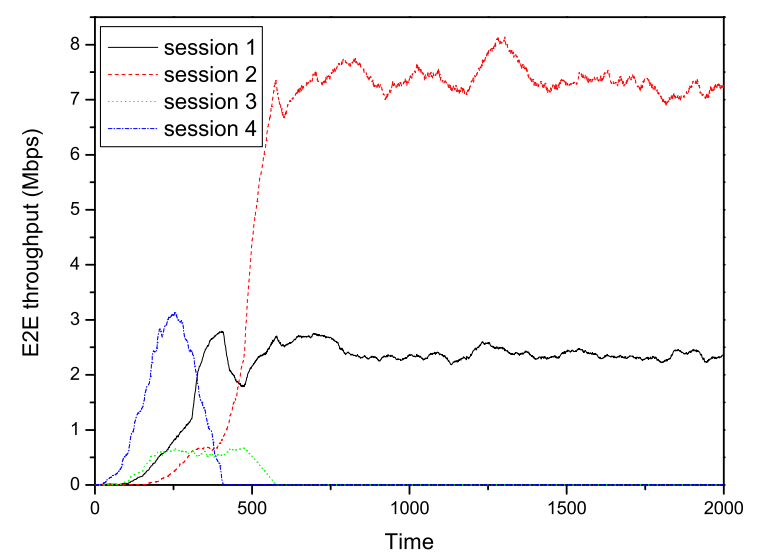

Fig. 10. E2E throughput in case of insufficient network capacity with a drop mechanism

control is necessary. Estimating the network capacity is in general a difficult problem [22][23][24], but we can estimate the network congestion according to the queue sizes in the network, especially the sizes of the overflow queues in the source nodes. For example, if the size of an overflow queue increases with a speed larger than $\frac{1}{2} r_{f}$ for consecutive 20 time slots, the flow's requirement is not met, so this flow should be dropped to return its network resources.

If the network capacity is not large enough to transmit all traffic, some adjustment at the sources of each session is necessary. The adjustment method depends on the properties of the sessions' services. When the overflow queue is too large at the traffic sources, the source nodes should decrease the data rate into the network, if possible, or reject some sessions if the throughput requirements are strict.

Figs. 9 and 10 show the performance comparison of the cases with or without session drop process. The simulation configuration is the same as in Section V.B, except the bandwidth of each channel is $0.1 \mathrm{Mbps}$. Without any drop mechanism, only session 1 can achieve the required throughput. 
When a drop mechanism is deployed, the required throughputs of both session 1 and session 2 are provided by dropping session 3 and 4.

\section{PRACTICAL ISSUES}

We now discuss some practical issues associated with the proposed scheme.

Data remaining in the network: The queue-balancing method allows a certain portion, $\epsilon$, of the data to stay in the network for a long time or forever. Therefore, this method is suitable only for long-lived sessions. Channel coding is a way to correct the lost information. Interleaving can avoid bursty continuous errors to ensure the performance of data-correctness approaches [20].

Estimation of power masks: CR nodes can obtain the threshold $Q_{i k}^{L}$ in several ways. If the primary receiver is a transmitter as well, $Q_{i k}^{L}$ can be obtained based on the received signal strength of primary users. For a dummy primary receiver, the power mask can be estimated by restricting the interference at the edge of the service range of the primary transmitter. For example, the IEEE 802.22 Working Group [21] proposed a power-masking scheme by considering the distance from a CR node to the TV transmitter.

Estimation of $L$ : In order to prevent the data of a session from spreading to all the nodes of the network, the maximum number of hops from the sources to the destinations should be restricted. The author of [16] provided a method for finding $L$ heuristically by trying different values.

Limited buffer size: The required maximum queue size to guarantee the network stability is given for the proposed scheme. If the buffer size of each node is limited and not enough for the required value, the required capacity for network stability would be larger than that in our analysis. Not only the potential decrease but also the queue sizes in the limited buffers should be considered when making resource allocations.

\section{RELATED WORK}

In [14] and [24], the multi-commodity flow problem was investigated for wireless networks from an information-theoretic perspective. They adopt a combinational interference model to avoid interference between links, making the power control unaffect the performance of other nodes. Some bounds of the network capacity are derived.

Based on the multi-commodity flow model, several publications focus on the cross-layer resource-allocation problem in wireless networks. The authors of [6] investigated the routing and resource allocation in wireless networks. It is assumed that the link capacity is only a function of local resource allocation, but they did not consider the spectrum reuse at different links that are far apart from each other. In [5], routing, spectrum and power are controlled jointly for wireless networks. However, they control the spectrum allocation to find a conflict-free combination a priori. When the spectrum situation changes at any node in the network, this scheme must work again for the whole network, and the following routing and power control have to iterate from the initial state, which cannot work well for CR networks with spectrum dynamics.

For CR networks, there are only a few existing schemes for cross-layer joint resource allocation. In [25] and [26], centralized and distributed schemes are proposed for CR networks, respectively. Both of them only considered different available channels at each node for CR. By contrast, we considered more characteristics of CR. The power mask model is considered as a general case of the channel availability model. Spectrum dynamics, which are seldom analyzed in the previous research on resource allocation, are considered in this paper. With the queue-balancing flow control method, the proposed distributed resource-allocation scheme can deal with spectrum dynamics well by using local queue information only.

\section{CONCLUSIONS}

In this paper, the resource-allocation problem in multihop CR networks is modeled as a multi-commodity flow problem. To solve this problem, the queue-balancing flow control method is proposed. Considering the characteristics of $\mathrm{CR}$, we extend the queue-balancing to multi-hop CR networks with varying link capacity and dynamic spectrum conditions.

Using the queue-balancing framework, we analyzed distributed resource allocation. The data rate, power and channel allocation are determined by the local queue size and adjusted to reflect the status of channels and the throughput requirement on each link. The optimal rate control for each session on a link is derived first. Power allocation at nodes is divided into two levels, which are the power allocation between links and the water-filling power allocation for the channels within a link. Coordination between links for channel allocation is achieved via some control messages according to the estimated channel holding utilities for each channel on each link. Based on the analysis on resource allocation, a node-based distributed algorithm is proposed for joint flow control and resource allocation. The parameters in the proposed scheme are configured to achieve the network stability in the asynchronous case. The performance degradation caused by the waiting for a channel switch is evaluated and compared with the synchronous case. Based on the queue-balancing scheme, the resource allocation for spectrum dynamics can be adjusted only by using local queue information. The adaptation to the spectrum dynamics is also investigated. In the insufficient network capacity cases, the drop mechanism can reject some of the session to satisfy the throughput requirement of the remaining sessions. For the implementation of the proposed scheme, we consider several practical issues as well.

\section{ACKNOWLEDGMENTS}

The work reported in this paper was supported in part by NSF under Grants CNS-0519498 and CNS-0721529, China Scholarship Council, Intel Corporation, Nokia Corporation and BUPT PhD Innovation Foundation. 


\section{REFERENCES}

[1] J. Mitola, G. Maguire, "Cognitive radio: Making software radios more personal", IEEE Personal Communications, vol. 6, no. 4, pp. 13-18, Aug. 1999

[2] S. Haykin, "Cognitive radio: Brain-empowered wireless communications", IEEE Journal on Selected Areas in Communications, vol. 23, no.2, pp. 201-220, Feb. 2005

[3] I.F. Akyildiz, W.Y. Lee, M.C. Vuran, S. Mohanty, "NeXt generation/dynamic spectrum access/cognitive radio wireless networks: A survey", Computer Networks Journal (Elsevier), vol. 50, no. 13, pp. 2127 2159, Sep. 2006

[4] C.T. Chou, S. Shankar N, H. Kim, K.G. Shin, "What and how much to gain by spectrum agility?", IEEE J. Sel. Areas in Commun., vol. 25, no. 3, pp. 576 - 588, Apr. 2007

[5] Y. Xi, E.M. Yeh, "Distributed algorithms for spectrum allocation, power control, routing, and congestion control in wireless networks", Proc. of ACM Mobihoc 2007, pp. 180 - 189, Sep. 2007

[6] L. Xiao, M. Johansson, S.P. Boyd, "Simultaneous routing and resource allocation via dual decomposition", IEEE Trans. Commun., vol. 52, no. 7, pp. 1136 - 1144, Jul. 2004

[7] R.K. Ahuja, T.L. Magnanti, J.B. Orlin, "Network flows: Theory, algorithms and applications", Prentice-Hall, 1993

[8] C. Peng, H. Zheng, B. Y. Zhao, "Utilization and fairness in spectrum assignment for opportunistic spectrum access", ACM MONET, vol. 11, no. 4, pp. 555 - 576, May 2006

[9] Y. Yuan, P. Bahl, R. Chandra, T. Moscibroda, Y. Wu, "Allocating dynamic time-spectrum blocks in cognitive radio networks", Proc. of ACM Mobihoc 2007, pp. 130 - 139, Sep. 2007

[10] Y. Shi, Y.T. Hou, "Optimal power control for multi-hop software defined radio networks", Proc. IEEE Infocom 2007, pp. 1694 - 1702, May 2007

[11] K. Hamdi, W. Zhang, and K. B. Letaief, "Power control in cognitive radio systems based on spectrum sensing side information," Proc. IEEE ICC 2007, pp. 5161 - 5165, June 2007

[12] R. Pal, "Efficient routing algorithms for multi-channel dynamic spectrum access networks", Proc. IEEE DySPAN 2007, pp. 288-291, Apr. 2007

[13] G. Cheng, W. Liu, Y. Li and W. Cheng, "Joint on-demand routing and spectrum assignment in cognitive radio networks", Proc. IEEE ICC 2007, pp. 6499-6503, Jun. 2007

[14] R. Madan, D. Shah, O. Leveque, "Product multicommodity flow in wireless networks", submitted to IEEE Trans. Information Theory, 2006

[15] B. Awerbuch, T. Leighton, "A simple local-control approximation algorithm for multicommodity flow", Proc. of IEEE FOCS 1993, pp. 459 468, Nov. 1993

[16] B. Awerbuch, T. Leighton, "Improved approximation algorithms for the multi-commodity flow problem and local competitive routing in dynamic networks", Proc. of ACM STOC 1994, pp. 487 - 496, 1994

[17] R. Cruz, A. Santhanam, "Optimal routing, link scheduling and power control in multi-hop wireless networks", Proc. IEEE Infocom 2003, pp. 702-711, Mar. 2003

[18] G. Song, Y. Li, "Cross-layer optimization for OFDM wireless networks part I: theoretical framework", IEEE Trans. Wireless Commun., vol. 4, no. 2. pp. 614 - 624, Mar. 2005

[19] M.R. Garey, D.S Johnson, "Computers and intractability: A guide to the theory of NP-completeness", 1990

[20] J. Proakis, "Digital communications (4th edition)", McGraw Hill Higher Education, 2000

[21] IEEE 802.22 workgroup, "22-06-0003-02-0000_ ETRI-FT-I2R-Motorola-Philips-Samsung-Thomson_MAC_Spec"

[22] P. Gupta, P.R. Kumar, "The capacity of wireless networks", IEEE Trans. Information Theory, vol. 46, no. 2, pp. 388 - 404, Mar. 2000

[23] M. Kodialam and T. Nandagopal, "Characterizing the capacity region in multi-radio multi-channel wireless mesh networks", Proc. of ACM Mobicom 2005, pp. 73 - 87, Aug. 2005

[24] O. Leveque, R. Madan, D. Shah, "Uniform multi-commodity flow in wireless networks with gaussian fading channels", Proc. of IEEE ISIT 2006, pp. 1846 - 1850, July 2006

[25] Y.T. Hou, Y. Shi, H.D. Sherali, "Spectrum sharing for multi-hop networking with cognitive radios", IEEE J. Sel. Areas in Commun., vol. 26, no. 1, pp. 146 - 155, Jan. 2008

[26] Y. Shi, Y.T. Hou, "A distributed optimization algorithm for multi-hop cognitive radio network", Proc. of IEEE Infocom 2008, to appear, Apr. 2008

\section{APPENDIX A \\ PROOF OF THEOREM 3}

To prove the network stability, meaning that the queue sizes for each session at all nodes are finite, the basic idea is the total potential function decreases if the queue size is large enough. When the potential function is finite, the queue size must be finite.

In the resource-allocation process at every time slot, there are three parts that change the potential of the network.

1) Add $(1+\epsilon) r_{f}$ data into the network from the sources.

2) Balance the queues for each session at each node.

3) Transmit data through links to decrease the potential and remove the data out of the network from the corresponding queues at the destinations.

For the first part, because the potential function is a monotonous increasing convex function, the increase of the potential of session $f$ is at most $(1+\epsilon) r_{f} \phi_{f}^{\prime}\left(s_{i}\right)$, where $s_{i}$ is the source queue size after adding the traffic.

For the second part, balancing the queues for each session will never increase the potential because of the exponential potential function.

For the third part, when the data rate of session $f$ over link $l$ is $x_{l f}$, considering the error of the queue size at the receiver $e_{l f}$, the potential decrease $\delta_{l f}$ is

$$
\begin{aligned}
\delta_{l f}= & \phi_{f}\left(q_{l f, T}\right)-\phi_{f}\left(q_{l f, T}-x_{l f}\right) \\
& +\phi_{f}\left(q_{l f, R}+e_{l f}\right)-\phi_{f}\left(q_{l f, R}+e_{l f}+x_{l f}\right)
\end{aligned}
$$

Using Taylor series to decompose $\phi_{f}\left(q_{l f, T}\right)$ and $\phi_{f}\left(q_{l f, R}+\right.$ $\left.e_{l f}+x_{l f}\right)$, the above expression can be bounded further as

$$
\begin{aligned}
\delta_{l f} \geq & x_{l f} \phi_{f}^{\prime}\left(q_{l f, T}\right)-x_{l f}^{2} \phi_{f}^{\prime \prime}\left(q_{l f, T}\right) \\
& -x_{l f} \phi_{f}^{\prime}\left(q_{l f, R}+e_{l f}\right)-x_{l f}^{2} \phi_{f}^{\prime \prime}\left(q_{l f, R}+e_{l f}+x_{l f}\right) \\
\geq & x_{l f} \phi_{f}^{\prime}\left(q_{l f, T}\right)-x_{l f}^{2} \phi_{f}^{\prime \prime}\left(q_{l f, T}\right) \\
& -x_{l f} \phi_{f}^{\prime}\left(q_{l f, R}\right)-x_{l f} e_{l f} \phi_{f}^{\prime \prime}\left(q_{l f, R}+e_{l f}\right) \\
& -x_{l f}^{2} \phi_{f}^{\prime \prime}\left(q_{l f, R}+e_{l f}+x_{l f}\right)
\end{aligned}
$$

Consider the potential decrease along a path, the size of the queue at the receiver of one link is equal to that at the transmitter of the next hop link, because the queue sizes are balanced for each session at each node. This way, $x_{l f} \phi_{f}^{\prime}\left(q_{l f, R}\right)$ can cancel $x_{l f} \phi_{f}^{\prime}\left(q_{l f, T}\right)$ of the next hop link. Define $q_{f j}^{\max }$ as the maximum value of the sizes of the queues along path $j$, and $\mathscr{L}(j)$ as the link set of path $j$. The total potential decrease along the path is at least

$$
\begin{aligned}
& x_{l f} \phi_{f}^{\prime}\left(q_{f j}^{\max }\right)-x_{l f} \phi_{f}^{\prime}(0)-\sum_{l \in \mathscr{L}(j)} x_{l f}^{2} \phi_{f}^{\prime \prime}\left(q_{l f, T}\right) \\
& -\sum_{l \in \mathscr{L}(j)} x_{l f} e_{l f} \phi_{f}^{\prime \prime}\left(q_{l f, R}+e_{l f}\right) \\
& -\sum_{l \in \mathscr{L}(j)} x_{l f}^{2} \phi_{f}^{\prime \prime}\left(q_{l f, R}+e_{l f}+x_{l f}\right)
\end{aligned}
$$

The path $j$ has the maximum length $L$. Only considering the path from the node with the largest queue size to the 
destination, Eq. (36) can be bounded by

$$
\begin{aligned}
& x_{l f} \phi_{f}^{\prime}\left(q_{f j}^{\max }\right)-x_{l f} \phi_{f}^{\prime}(0)-2 L x_{l f}^{2} \phi_{f}^{\prime \prime}\left(q_{f j}^{\max }+x_{l f}\right) \\
& -\sum_{l \in \mathscr{L}(j)} x_{l f} e_{l f} \phi_{f}^{\prime \prime}\left(q_{l f, R}+e_{l f}\right)
\end{aligned}
$$

The upper bound of the error of queue size is $\frac{q_{f}^{\max }-q_{l f, R}}{4}$ as Eq. (29).

$$
\begin{aligned}
& x_{l f} e_{l f} \phi_{f}^{\prime \prime}\left(q_{l f, R}+e_{l f}\right) \\
= & x_{l f} \frac{q_{f}^{\max }-q_{l f, R}}{4} \alpha_{f}^{2} e^{\frac{3}{4} \alpha_{f} q_{l f, R}} e^{\frac{1}{4} \alpha_{f} q_{f}^{\max }}
\end{aligned}
$$

In order to obtain the performance in the worst case, the firstorder derivative of Eq. (38) with respect to $q_{l f, R}$ is

$$
x_{l f} e^{\left(\frac{1}{4} q_{f}^{\max }+\frac{3}{4} q_{l f, R}\right) \alpha_{f}}\left(-\frac{1}{4}+\frac{3}{4} \alpha_{f} \frac{q_{f}^{\max }-q_{l f, R}}{4}\right)
$$

The performance is worst when the derivative is equal to 0 . Solving this equation, we can obtain the solution as

$$
q_{l f, R}=q_{f}^{\max }-\frac{4}{3 \alpha_{f}}
$$

Substituting Eq. (40) into Eq. (38), we get

$$
x_{l f} e_{l f} \phi_{f}^{\prime \prime}\left(q_{l f, R}+e_{l f}\right) \leq x_{l f} \frac{1}{3 \alpha_{f}} \phi_{f}^{\prime \prime}\left(q_{f}^{\max }-\frac{1}{\alpha_{f}}\right)
$$

Suppose the network can transmit $(1+2 \epsilon) r_{f}$ data for each session $f$. Partition the flow for each session $f$ into several paths, which has the E2E throughput $x_{f, j}$ for path $j$ of session $f$. Let $q_{f, j}^{\max }$ be the maximum queue size for session $f$ along path $j$. The total potential decrease in the network is

$$
\begin{aligned}
& \sum_{f} \sum_{l} x_{l f}\left(\phi_{f}^{\prime}\left(q_{l f, T}\right)-\phi_{f}^{\prime}\left(q_{l f, R}\right)\right) \\
& -x_{l f}^{2}\left(\phi_{f}^{\prime \prime}\left(q_{l f, T}\right)-\phi_{f}^{\prime \prime}\left(q_{l f, R}+e_{l f}+x_{l f}\right)\right) \\
& -x_{l f} e_{l f} \phi_{f}^{\prime \prime}\left(q_{l f, R}+e_{l f}\right) \\
\leq & \sum_{f} \sum_{j} \sum_{l} x_{f, j}\left(\phi_{f}^{\prime}\left(q_{l f, T}\right)-\phi_{f}^{\prime}\left(q_{l f, R}\right)\right) \\
& -2 x_{f, j}(1+2 \epsilon) r_{f} \phi_{f}^{\prime \prime}\left(q_{f, j}^{\max }+x_{f, j}\right) \\
& -x_{f, j} \frac{1}{3 \alpha_{f}} \phi_{f}^{\prime \prime}\left(q_{f, j}^{\max }-\frac{1}{\alpha_{f}}\right) \\
\leq & \sum_{f} \sum_{j} x_{f, j}\left(\phi_{f}^{\prime}\left(q_{f j}^{\max }\right)-\phi_{f}^{\prime}(0)\right) \\
& \left.-2 x_{f, j} L(1+2 \epsilon) r_{f} \phi_{f}^{\prime \prime}\left(q_{f j}^{\max }+(1+2 \epsilon) r_{f}\right)\right) \\
& -x_{f, j} L \frac{1}{3 \alpha_{f}} \phi_{f}^{\prime \prime}\left(q_{f j}^{\max }-\frac{1}{\alpha_{f}}\right)
\end{aligned}
$$

When $\alpha_{f}=\frac{\epsilon}{16 L r_{f} \ln \frac{4 L}{3 \epsilon}}$,

$$
\begin{aligned}
& \left.2 L(1+2 \epsilon) r_{f} \phi_{f}^{\prime \prime}\left(q_{f j}^{\max }+(1+2 \epsilon) r_{f}\right)\right) \\
= & 2 L(1+2 \epsilon) r_{f} \alpha_{f} e^{\alpha_{f}(1+2 \epsilon) r_{f}} \phi_{f}^{\prime}\left(q_{f j}^{\max }\right) \\
\leq & \frac{\epsilon}{4} \phi_{f}^{\prime}\left(q_{f j}^{\max }\right)
\end{aligned}
$$

$$
\begin{aligned}
& L \frac{1}{3 \alpha_{f}} \phi_{f}^{\prime \prime}\left(q_{f j}^{\max }-\frac{1}{\alpha_{f}}\right) \\
= & \frac{L}{3} \frac{1}{e^{\frac{1}{\alpha_{f}}}} \phi_{f}^{\prime}\left(q_{f j}^{\max }\right) \\
\leq & \frac{\epsilon}{4} \phi_{f}^{\prime}\left(q_{f j}^{\max }\right)
\end{aligned}
$$

The configuration of $\alpha_{f}$ lets Eq. (43) and Eq. (44) be only a small fraction of $\phi_{f}^{\prime}\left(q_{f j}^{\max }\right)$.

The lower bound of the potential decrease in the network, Eq. (42), can be rewritten as

$$
\begin{aligned}
& \sum_{f} \sum_{j} x_{f, j}\left(\left(1-\frac{\epsilon}{2}\right) \phi_{f}^{\prime}\left(q_{f j}^{\max }\right)-\phi_{f}^{\prime}(0)\right) \\
\geq & \sum_{f}(1+2 \epsilon) r_{f}\left(\left(1-\frac{\epsilon}{2}\right) \phi_{f}^{\prime}\left(s_{f}\right)-\alpha_{f}\right) \\
\geq & \left(1+\frac{3}{2} \epsilon-\epsilon^{2}\right) \sum_{f} r_{f} \phi_{f}^{\prime}\left(s_{f}\right)-(1+2 \epsilon)|\mathscr{F}| r_{f} \alpha_{f}(45)
\end{aligned}
$$

Subtracting the potential increase caused by the entering data from Eq. (45), we can obtain the total potential decrease as

$$
\left(\frac{1}{2} \epsilon-\epsilon^{2}\right) \sum_{f} r_{f} \phi_{f}^{\prime}\left(s_{f}\right)-(1+2 \epsilon)|\mathscr{F}| r_{f} \alpha_{f}
$$

If session $f$ need to use the overflow at its source node, $s_{f}=q_{f}^{\max }$, the potential decrease is at least

$$
\left(\frac{1}{2} \epsilon-\epsilon^{2}\right) r_{f} \phi_{f}^{\prime}\left(q_{f}^{\max }\right)-(1+2 \epsilon)|\mathscr{F}| r_{f} \alpha_{f}
$$

Let Eq. (47) be equal to 0 and then solve the equation. We can obtain the value of $q_{f}^{\max }$ as Eq. (31). With these $q_{f}^{\max }$, as long as the overflow queue of one session has data, the potential decrease is nonnegative. The total potential of the network will not grow infinitely. Therefore, the parameter configuration in Theorem 3 can guarantee the network stability.

\section{APPENDIX B \\ PROOF OF THEOREM 5}

Based on the proof of Theorem 3, from Eq. (45), if the provided E2E throughput is $\xi$, then the potential decrease is

$$
\left(1-\frac{1}{2} \epsilon\right) \xi \sum_{f} r_{f} \phi_{f}^{\prime}\left(s_{f}\right)-(1+2 \epsilon)|\mathscr{F}| r_{f} \alpha_{f}
$$

Considering the potential increase at the sources of sessions, to let Eq. (48) be positive, it must satisfy

$$
\left(1-\frac{1}{2} \epsilon\right) \xi>(1+\epsilon)
$$

So, we can obtain that

$$
\xi>\frac{2+2 \epsilon}{2-\epsilon} r_{f}
$$

On the other hand, if $\xi<(1+2 \epsilon) r_{f}$, the maximum queue size in Theorem 3 is not large enough. Using a method similar to the proof of Theorem 3, $q_{f}^{\max }$ should be increased to

$$
q_{f}^{\max }=\frac{1}{\alpha_{f}} \ln \left(\frac{|\mathscr{F}|(1+2 \epsilon)}{\left(1-\frac{1}{2} \epsilon\right) \xi-(1+\epsilon)}\right)
$$

